UCRL-JC-124891

PREPRINT

\title{
The Use of Beam Propagation Modeling of Beamlet and Nova to Ensure a "Safe" National Ignition Facility Laser System Design
}

\author{
M. A. Henesian, P. Renard, J. Auerbach, \\ J. Caird, R. Ehrlich, S. W. Haney, J. Hunt, \\ J. Lawson, K. Manes, D. Milam, R. Sacks, \\ L. Seppala, I. Smith, R. Speck, C. Thompson, \\ B. VanWonterghem, P. Wegner, T. Weiland, \\ C. Widmayer, W. Williams, and J. B. Trenholme
}

This paper was prepared for submittal to the 2nd Annual International Conference on Solid-State Lasers for Applications to Inertial Confinement Fusion Paris, France October 22-25, 1996

March 17, 1997

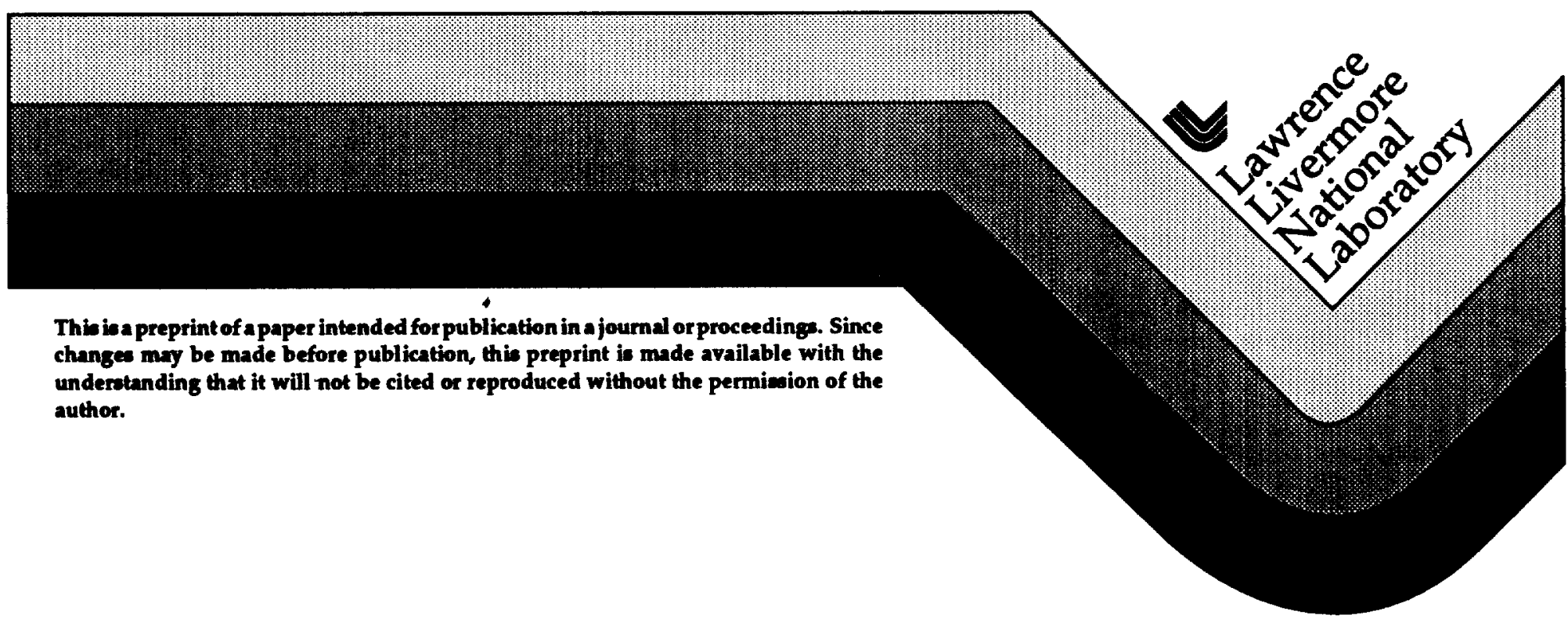




\section{DISCLAIMER}

This document was prepared as an account of work sponsored by an agency of the United States Government. Neither the United States Government nor the University of California nor any of their employees, makes any warranty, express or implied, or assumes any legal liability or responsibility for the accuracy, completeness, or usefulness of any information, apparatus, product, or process disclosed, or represents that its use would not infringe privately owned rights. Reference herein to any specific commercial product, process, or service by trade name, trademark, manufacturer, or otherwise, does not necessarily constitute or imply its endorsement, recommendation, or favoring by the United States Government or the University of California. The views and opinions of authors expressed herein do not necessarily state or reflect those of the United States Government or the University of California, and shall not be used for advertising or product endorsement purposes. 


\title{
The use of beam propagation modeling of Beamlet and Nova to ensure a "Safe" National Ignition Facility laser system design
}

\author{
M. Henesian, P. Renard, J. Auerbach, J. Caird, B. Ehrlich, S. Haney, J. Hunt, J. Lawson, K. Manes, \\ D. Milam, R. Sacks, L. Seppala, I. Smith, R. Speck, C. Thompson, B. VanWonterghem, \\ P. Wegner, T. Weiland, C. Widmayer, W. Williams, and J. B.Trenholme
}

\author{
Lawrence Livermore National Laboratory \\ P. O. Box 808 , L-490 \\ Livermore, CA $94551-9900$
}

\begin{abstract}
An exhaustive set of Beamlet and Nova laser system simulations were performed over a wide range of power levels in order to gain understanding about the statistical trends in Nova and Beamlet's experimental data sets, and to provide critical validation of propagation tools ${ }^{1}$ and design "rules" applied to the 192-arm National Ignition Facility (NIF) at Lawrence Livermore National Laboratory (LLNL). The experiments considered for modeling were at 220-ps FWHM duration with unpumped booster slabs on Beamlet, and 100-ps FWHM with pumped 31.5-cm and 46-cm disk amplifiers on Nova. ${ }^{2}$ Simulations indicated that on Beamlet, the $\Delta B$ (the intensity-dependent phaseshift parameter characterizing the tendency towards beam filamentation) for the booster amplifier stage without pumping, would be nearly identical to the $\triangle \mathrm{B}$ expected on $\mathrm{NIF}$ at the peak of a typical 20-ns long shaped pulse intended for ICF target irradiation. Therefore, with energies less than $1 \mathrm{~kJ}$ in short-pulses, we examined on Beamlet the comparable $\triangle B$-driven filamentation conditions predicted for long ICF pulseshapes in the $18 \mathrm{~kJ}$ regime on the NIF, while avoiding fluence dependent surface damage. Various spatial filter pinhole configurations were examined on Nova and Beamlet. Open transport spatial filter pinholes were used in some experiments to allow the direct measurement of the onset of beam filamentation. Schlieren images on Beamlet of the far field irradiance measuring the scattered light fraction outside of 33- $\mu$ radians were also obtained and compared to modeled results. ${ }^{3}$
\end{abstract}

A measure of beam modulation in simulations and experiments is the time-integrated contrast value, $\mathrm{C}$, defined as the square-root of the variance of the fluence normalized to the average. This was correlated to the $\Delta \mathrm{B}$ value calculated for the unpumped booster amplifier stage on Beamlet, or to Nova's 46-cm disk amplifier stage. The timedependent intensity contrast could be simulated, but was not measured. PROP92's unique optically-induced surface damage and intensity-dependent beam filamentation codes were activated for these simulations. ${ }^{4}$ PROP92's damage predictions with unpumped booster slabs correlates very well with the measured and predicted sharp increase in contrast $C$ beyond about $10 \%$, at $\Delta B$ values of $1.8 \sim 2.4$ radians, depending on pinhole configuration. For $\Delta B$-values much above 2.2-radians with $200-\mu$ radian cavity and transport filter pinholes, or 2.8-radians with 130- $\mu$ radian pinholes, we expect to observe filamentation-induced damage in the last booster amplifier disks and/or input spatial filter lens. With an "open" transport filter pinhole, damage would be expected in the output spatial filter lens and other down stream optics. Therefore, for "safe" operation, with margin to be determined, the $\Delta \mathrm{B}$ should be limited to 1.8 to 2.4 radians, depending on spatial filtering, corresponding to a fluence contrast of $10 \%$ or less. Applying the contrast and $\Delta \mathrm{B}$ limits to NIF requires detailed system simulations.' The measured increase in scattered far field energy outside of $33 \mu$ radians at $\Delta B$ 's above $\sim 1.8$ radians is consistent with predictions and indicates the onset of undesirable nonlinearities.

Keywords: optical damage, beam filamentation, self-focusing, Schlieren, Beamlet, Nova, NIF 


\section{INTRODUCTION}

Evidence of small-scale self-focusing (i.e., beam filamentation) damage in the amplifier slabs and spatial filter lenses on both Beamlet and Nova, repeated damage to the input lens of the final spatial filter on Nova following the Nova 46-cm disk amplifiers, and filamentation in the $3 \omega$ target chamber optics on Nova have led us to conclude that the beam irradiance in the final stages of these systems is unexpectedly high. The observed bulk damage would require an irradiance in excess of $100 \mathrm{GW} / \mathrm{cm}^{2}$. Near-field data on film or CCD cameras suggest that even under stressful operating conditions, the peak-to-average modulation is typically only 1.5 to 1 , resulting in far less irradiance than required for beam filamentation. It is very likely that diagnostics measuring the integrated near-field exposures for long pulses, mask high irradiance conditions through an averaging process. Indeed, potentially high, peak irradiances would be expected at the end of a long, saturating pulse at precisely the point where peak B-integral conditions are experienced. To examine this, short-pulse experiments were performed on Beamlet and Nova to approximate the conditions at the end of a long saturating pulse. ${ }^{2}$ Data was analyzed with the goal of determining safe operating conditions to preclude filamentation damage on Beamlet and Nova. Validation of the modeling for Beamlet.and Nova, then allowed us to establish "safe" design and operational guidelines for those systems and for the NIF, through detailed propagation analysis.

\section{EXPERIMENTS}

Beamlet uses an 11-0-5 amplifier architecture with 11 slab amplifiers in a 4-pass cavity, a large aperture plasmaelectrode Pockels cell, and 5 booster slabs that in most ways is proto-typical of one arm of the NIF. ${ }^{5}$ Fig. 1 shows a schematic of the Beamlet 11-0-5 architecture. On the NIF, each of the nearly identical 192 laser arms will use this design, except for a $23.5-\mathrm{m}$ long cavity spatial filter and a $60-\mathrm{m}$ long transport spatial filter, in order to provide image relaying to the frequency conversion crystals and $3 \omega$ focusing optics located 66.5 meters from the transport filter output lens. On Beamlet, the cavity and transport filters are both $18-\mathrm{m}$ long. We conducted experiments on Nova and Beamlet to simulate the stressful conditions at the end of high energy pulses used for target irradiation. This type of operation poses a high damage threat since the largest B-integral value occurs during a brief interval near the end of such pulses. During high fluence extraction, amplifier gain significantly decreases during the pulse because the early portions extract significant energy from the amplifiers. To produce the desired output shape, this effect must be offset by shaping the injected pulse so that its irradiance increases monotonically with time. Since the B-integral is proportional to the irradiance, it also increases during the pulse. Therefore, the highest damage threat from nonlinear growth of modulation occurs at the end of the pulse where the amplifier gain is lowest and the Bintegral is highest.

To mimic this effect, we used short pulse lengths and reduced amplifier gains. The pulses were Gaussian in shape with duration's of $220 \mathrm{ps}$ on Beamlet and $100 \mathrm{ps}$ on Nova. The gains of the amplifiers were reduced to the gain seen by the last photon in long energetic pulses. On Nova, the amplifier gains were set to imitate those at the end of a 1 ns flat output pulse with an energy of $8 \mathrm{~kJ}$ at $8 \mathrm{TW}$, a typical Nova shot. The multipass architecture of Beamlet makes gain adjustment difficult because reducing the gain of the cavity amplifiers affects all four passes. Therefore, Beamlet tests were conducted with the cavity section fully pumped and the booster section tumed off. This arrangement gives a lower $\triangle B$ in the cavity and higher $\triangle B$ in the booster than normal 200-ps Beamlet operation, but the $\triangle \mathrm{B}$ in the booster section would then be very comparable between Beamlet and NIF. We illustrate this in Fig. 2, where the B-integral value as a function of position in the amplifier chain is shown on the left for Beamlet, and on the right for a NIF design. On Beamlet (without booster amplification), a 3-TW/200-ps pulse and an $18 \mathrm{~kJ}$ shaped ICF target physics pulse on NIF, give nearly the same peak $\Delta \mathrm{B}$ of 1.8 to 1.9 radians in the booster amplifier slabs. 
Spatial filter pinhole configurations were varied to assess the effect of tighter spatial filtering on the quality of the beam. On Nova, we used two different pinholes in both the final (SF7) and penultimate (SF6) spatial filters. In SF6, pinhole diameters were $1.5 \mathrm{~mm}$ which passes angles of $100 \mu$ radians, and $3 \mathrm{~mm}$ which passes $200 \mu$ radians. In SF7, pinhole diameters were $1.8 \mathrm{~mm}$ ( $100 \mu$ radians acceptance angle) and $4 \mathrm{~mm}$ ( $220 \mu$ radians). On Beamlet we used pinhole angles of 130,150, and $200 \mu$ radians in the cavity, and 100,130,150, $200 \mu$ radians and the "open position" in the transport filter. By opening the transport spatial filter pinhole we can image the beam at the input lens $\mathrm{L} 3$ with high resolution. To increase the resolution of the $1 \omega$ output diagnostics near-field camera on Beamlet, we installed a system with an angular acceptance up to $5 \mathrm{mrad}$ (referenced to main beamline). The magnification of the system was 17.6, so each 13.5 micron square pixel of the $1 \mathrm{~K}$ x $1 \mathrm{~K}$ pixel CCD camera corresponded to $240 \mu \mathrm{m}$ in the main beam. The size of the object plane was $24 \times 24 \mathrm{~cm}$. The estimated optical resolution was $300 \mu \mathrm{m}$ in the full size beam. Nova also has a high resolution near-field camera in its output sensor that records an image passing through Schott RG 1000 filter glass onto an $8 \times 10$ inch sheet of Kodak Tri-X film. The device was adjusted to capture beam profiles in the image plane of the transport spatial filter (SF7) input lens. All data was normalized to the $44 \mathrm{~cm}$ diameter beam. The magnification of the film image relative to the $44 \mathrm{~cm}$ beam was 0.459 . Film data was densitometered using $200 \mu \mathrm{m}$ square slits, giving a resolution of $0.436 \mathrm{~mm}$ per pixel. The film was calibrated from exposures of a standard set of calibration spots, and multiple sets were used to devise an average density-toexposure curve to obtain near-field intensities. Film development was consistent over the course of these experiments, yielding density vs. exposure curves that showed $10 \%$ reproducibility.

A Schlieren experiment was also set-up on Beamlet to examine scattered light content outside of 20,33 , and 66$\mu$ radians. ${ }^{3}$ Instead of the conventional beam block at a focal plane, our diagnostic used a "scraper" mirror to separate the central focal region from the regions of interest. After a sample of the incident output sensor diagnostic beam was split and collected in an energy meter, the beam was focused onto a spherical mirror that had a small circular hole drilled in its center. Light passing through the hole was monitored by a CCD camera, as an alignment aid, and collected in an energy meter for use in balance calculations. Light reflected from the mirror was collected onto near field and far field CCD camera systems and onto more energy meters. The angular acceptance of the diagnostic was $800-\mu$ radians (half-angle). Only data from shots that were well aligned with the Schlieren mirror hole were accepted for analysis.

\section{PROPAgATION MODELING}

The computer codes to model beam propagation are sophisticated assemblages of algorithms using perturbation techniques and spectral analysis. ${ }^{4}$ Diffraction effects, whole-beam effects, gain saturation, temporally resolved surface damage models, filamentation (bulk damage) models, deformable mirror and other phase-inducing numeric models for spatial filter pinholes, are all united to simulate laser pulse propagation through complicated optical chains, predicting the field evolution of the pulse as well as material response of the various optical components. The propagation code PROP92 at LLNL was used pre- and post-experiment to model the conditions observed during these experiments. Previously, PROP92 was validated by modeling laboratory experiments to investigate the selffocusing of a $1 \omega$ beam in a long fused silica sample obscured upstream by a thin wire. ${ }^{6}$ Calculations were also performed using BT-GAIN, which has been validated by modeling laboratory demonstrations of nonlinear 4-wave mixing and conjugated "hot spot" imaging around circular obscurations.' By adjusting various input parameters in these simulation codes, calculated data can be generated that spans the typical clusters of experimentally obtained data. More importantly, calculated or modeled parameters can be scaled to determine the efficacy of the model in light of experimental evidence.

A critical input to the modeling calculations is the short scale length phase noise distributions assigned to the optical components, especially the amplifier disks and spatial filter lenses. We enter phase noise on the optics in 
calculations as Power Spectral Density (PSD) that is derived from high resolution interferometry of the optical parts. 7 The PSD is a measure of the phase retardation of a transmissive optic (or alternately, the phase retardation due to the surface contour of a reflective optic) in terms of its spatial frequencies. While the PSD has the advantage of being a real quantity that expresses the spectral distribution of the wavefront error, it does not possess any relative phase information in the Fourier domain. Thus, simulations based on the PSD do not uniquely specify a given wavefront, but instead an ensemble of wavefronts, each possessing similar spatial frequencies without necessarily being identical. However, PSD based aberration files have the identical phase auto-correlation distribution as the real data from which they were derived.

Over the last year, a data base of phase noise distributions has been compiled for the Beamlet amplifier slabs but very little for Nova amplifier disks. Fortunately we were able to use the Beamlet database, as interferometer measurements showed that the phase noise distributions of a Nova amplifier disk were very similar to those for Beamlet slab 19. Slab type 19 is characterized by a 4.62 -nm r.m.s. total transmitted wavefront error with 1.49 , 2.17, and 3.80-nm r.m.s. distributed over the ranges from 0.5 to $6 \mathrm{~mm}, 6 \mathrm{~mm}$ to $33 \mathrm{~mm}$, and greater than $33 \mathrm{~mm}$ scale lengths, respectively. We were able to get good agreement with Nova data by using Beamlet slab 19 phase noise data with power scaled up by a factor of 2.25 . In addition to amplifier disk phase noise, the simulations also accounted for spatially varying gain and apodization of the beam, including outer edge profiles and, in the case of Nova, the central vertical strip apodizer for the split-disk amplifiers. The Nova calculations modeled the 31.5 and 46-cm disk amplifier sections and spatial filters up to powers of 10-TW (or $\triangle B$ of 5 radians in 46-cm amplifier stage).

For the Beamlet modeling, simulations were performed up to $7 \mathrm{TW}$ power ( $\Delta \mathrm{B}$ up to 3.63 radians) with $130-\mu \mathrm{R}$ pinholes, and up to $5-T W$ power ( $\triangle B$ up to 2.60 radians) with 200- $\mu R$ cavity pinholes. The beam size was $34 \times 34$ $\mathrm{cm}$ at the $1 \%$ power level. An end-to-end model for the Beamlet system, beginning with the beam shaping apodizers, including the 4-pass rod amplifier, front-end telescope systems, and deformable mirror was constructed; and as it turns out was essential for the simulations to fit the various data sets, with pinhole and power variations. Based on the Beamlet amplifier slab type-16, simulated phase aberration files were used throughout these nuns with no power scale-up factor. Slab type 16 is characterized by a 3.84-nm r.m.s. transmitted wavefront error with 1.19, 1.49 , and 3.33-nm r.m.s. distributed over the ranges from 0.5 to $6 \mathrm{~mm}, 6 \mathrm{~mm}$ to $33 \mathrm{~mm}$, and greater than $33 \mathrm{~mm}$ scale lengths, respectively. Simulated phase files for the small components in the front-end, the cavity and transport spatial filter lenses, the Pockel's cell crystal, and amplifier disks were taken from the component data base." The Pockel's cell data was rotated through 90 degrees so that the diamond-tuned diffraction lines would be near vertical, as they appear on Beamlet. Low power (1 TW) runs were performed to determine the 39-actuator deformable mirror correction' and also to extract the input beam shape to give a nominally flat output. Figure 3 shows the simulated and measured profiles for the beam injected into the main 4-pass cavity of Beamlet. The predicted contrast of 4.78 $\%$ is in excellent agreement with the measured contrast of $4.55 \%$. This provides a lower limit to the fluence contrast of the Beamlet system.

Spatial resolution in the simulations was varied to provide a self-consistency check. Typically, 512 x 512 points were used on a 50-cm computational grid, but some checking was done at $1 \mathrm{~K} \times 1 \mathrm{~K}$ resolution under conditions of high beam modulation. Comparison with a $1 \mathrm{~K}$ point run (each takes $\sim 13 \mathrm{hrs}$.) at $5 \mathrm{TW}$ power with 200 uradian cavity and transport pinholes indicated that the 512-point run over-estimates the contrast at the input spatial filter lens $\mathrm{L} 3$ by $\sim 16 \%$, but under-estimates the contrast by $\sim 8 \%$ at the output relay plane. The $99 \%$ modulation value is over-estimated by $\sim 20 \%$ at $L 3$, and under estimated by $8 \%$ at the output plane. At high $\Delta B$ (over 2.4 radians) and high contrast $(\mathrm{C}>0.25)$ at $\mathrm{L} 3$ with $200 \mu$ radian pinholes, the standard 512 point runs become aliases as they do not have adequate spatial resolution in components where the beam begins to develop filamentation. As another check, a 
$1 \mathrm{~K}$ point run was performed at $5 \mathrm{TW}$ with $130-\mu \mathrm{R}$ cavity and transport pinholes. The higher resolution run gave an $11 \%$ higher peak intensity value at L3, but the contrast and F99\% numbers were very close at $\mathrm{L} 3$ and the output plane. Temporal resolution was increased from 5 to 7 time-slices at $512 \times 512$ points, but for contrasts of $25 \%$ (0.25) or less, it appeared that five time slices was sufficient to guarantee proper nonlinear treatment of the high intensity portions of the beam.

\section{DATA REDUCTION}

Standard statistical methods were applied to analyze both experimental and simulated data. The near-field intensity profiles were analyzed at selected positions along the propagation path, varying observed peak powers (and hence $\Delta \mathrm{B}$ ) for a fixed set of pinholes. Near-field images were analyzed by extracting small $5 \times 5 \mathrm{~cm}$ subregions from the overall beam image and conducting a statistical analysis on the patch. The subimage was chosen to avoid full beam edge effects and to obtain a sample of the beam with relatively constant background irradiance. From the simulations, having fewer available pixels but no artifacts, we extracted a $15 \times 15 \mathrm{~cm}$ patch from the centers of each fluence profile and performed the same statistical analysis. For each data patch a histogram was constructed giving the number of pixels occurring at each fluence. The histograms are normalized so that the area under each curve is equal to one. The shape of the histogram depends on the power, which is linearly proportional to the $\Delta \mathrm{B}$ value for the booster slabs or disks. At low power the spatial profile of the beam is generally quite uniform and the histogram tends to be narrow and sharply peaked about the average fluence. As power increases, beam quality degrades; i.e., the irradiance modulation of the beam grows and the histogram tends to broaden, decrease in amplitude, and develop a noticeable skew towards higher intensity. ${ }^{2}$

We found that the most robust figure of merit for assessing beam quality below and at the onset of beam filamentation was the second moment of the histogram, which measures the width, or the variation of the histogram about its mean. The contrast ratio $C$ is defined to be: $C=\operatorname{sqrt}\left(\sum_{(i=1, N)}\left(F_{1}-F_{\text {avg }}\right)^{2}\right) / F_{\text {avg }}$, where $F_{\text {avg }}$ is the average fluence of the patch analyzed, $N$ is the number of pixels in the patch, and $F_{i}$ is the fluence of each pixel. This quantity was calculated for each patch analyzed. As a measure of the peak modulation, we also calculated the $99^{\text {th }}$ percentile, $F_{99}$, which is the fluence (in units of $F_{a v z}$ ) below which $99 \%$ of the pixels occur. At moderate contrasts, $\mathrm{C}<0.1, \mathrm{~F}_{99}=1+2.3 \mathrm{C}$ since the fluence histogram resembles a Gaussian distribution.

\section{BEAMLET COMPARISON}

We calculated beam statistics from the Beamlet shot data and compared them to computer simulations for four pinhole configurations. The pinhole acceptance angles (uradians) for the cavity and transport filters in each case were: 130/open, 130/130, 200/open, and 200/200 respectively. Figures 4 (left, right sides) shows the contrast ratio $C$, as a function of the booster section's B-integral for the 200/open and 130/open pinhole configurations. Figures 5 (left, right sides) shows the corresponding fluence modulation. The solid squares are the results of the computer simulations and the shot data are represented by the circles. The error bars shown are $\pm 15 \%$ for the contrast and $\pm 10 \%$ for modulation. Due to beam nonuniformity, some data showed larger variation than this. The agreement with experimental trends is good. It appears that we may be over-estimating the contrast and modulation at $\Delta \mathrm{B}>2.5$ radians for 200/open case (Figs. 4), and under-estimating the experimental quantities for the 130/open case (Figs. 5). Note that the rate of change of the contrast ratio is quite gradual for low values of B-integral but increases dramatically at high $B$ values. The rate of increase is much steeper with the larger cavity pinholes and indicates an increased optical damage risk, particularly with nonuniform beams. Earlier simulations that did not include the front-end model required that the disk amplifier slab noise be increased in power by three-times in order to fit the 130/open data. This caused the contrast prediction of the 200/open data to lie well above experimental points at low 
power, and rise more rapidly than the data at high power. Including the noise injected from the front-end has brought the data at 130/open and 200\%open into better agreement.

To control beam modulation down stream of the transport filter requires a tight pinhole in the filter. Figures 6 (left, right sides) shows the contrast data and predictions for the 200/200 configuration (left) and 130/130, and

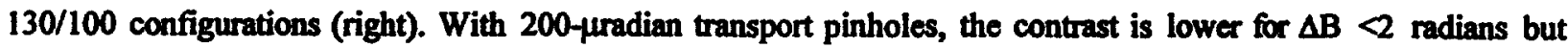
still rises quite rapidly afterwards. The tight $100-\mu$ radian transport pinhole (right side, Fig. 6 ) controls the modulation over a wide power range (up to $\triangle \mathrm{B} \sim 3$ radians, or equivalently $5.8 \mathrm{TW}$ power). However, further

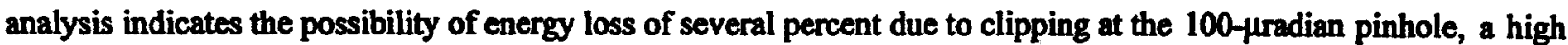
sensitivity to pinhole misalignment, and the possibility of increased modulation due to the "Gibbs" effoct at the pinhole (Fourier transform) plane.

Figure 7 shows a collection of the Schlieren energy data mentioned earlier. We plot data for 130/open, 130/100, 200/open, 200/200 pinhole configurations, and values obtained from high dynamic range 10 far field measurements

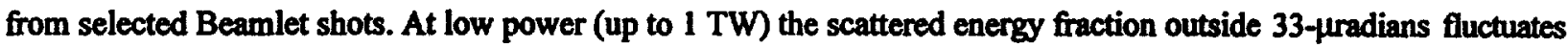
between 1 and $2 \%$, but begins to rise with power up to $5 \sim 8 \%$ at $4 \mathrm{TW}$. Simulations predict a more rapid rise with the larger cavity pinhole configurations, but the data shows higher scattered energy with the $130 / 100 \mu$ radian pinholes. Experimental difficulty and large data set variations (not all data shown here) indicates that we should not draw too many conclusions. However, at powers above about $4 \mathrm{TW}(\Delta \mathrm{B} \sim 2$ radians), as the contrast measurements indicate a rapid rise in fluence modulation, the Schlieren data sets also indicate the onset of increased light scatter, which is consistent with the nonlinear growth of intensity-dependent spatial phase modulation and beam filamentation (self-focusing).

\section{NOVA COMPARISON}

As in the Beamlet case, we calculated beam statistics from Nova data and compared the results to simulations for four pinhole configurations. The Nova beam contains an additional feature: pronounced long scale length intensity fluctuations in the aperture. Obtaining a record of the noise sources for Nova was difficult as we had phase measurements of only one $31 \mathrm{~cm}$ disk. Since this measurement yielded characteristics similar to Beamlet's slab 19 interferometric data, we used the latter with power scaled by 2.25 for matching data for all amplifiers in the Nova simulations.

Figure 7 (left side) shows the contrast ratio plotted against the B in the $46 \mathrm{~cm}$ section for the Nova standard $200 / 200 \mu$ radians pinhole configuration as well as the 100/200 $\mu$ radians case. Note: $B$ in this figure was computed from the irradiance at the input lens to the final (SF7) filter, ignoring any pinhole effects in SF7. However, the pinhole was present and energy loss was noted in experiments at the higher irradiances. This graph suggests that unlike Beamlet, Nova can tolerate higher values of $B$ before the onset of rapid beam degradation. We attribute this higher threshold to the filtering action of SF7. The agreement between data and simulations that use the scaled noise sources is quite good. In particular, it is apparent that tighter filtering in the penultimate filter (SF6) extends the threshold for beam degradation.

Figure 7 (right side) shows comparisons of two other pinhole configurations: 100/100 $\mu$ radians and 200/100 $\mu$ radians. In this figure, simulations are failing to match experimental data. Indeed, it is somewhat counter-intuitive that the tighter filtering in both the penultimate (SF6) and final (SF7) filters appears to make the beam degrade sooner. The $200 / 100 \mu$ radians data is more in line with our current understanding of the filtering process. Further tests to measure the noise sources entering the final stages on Nova may provide some insight into these phenomena. The current standard pinhole configuration is $200 \mu$ radians for Spatial Filter 6 's output and 200 


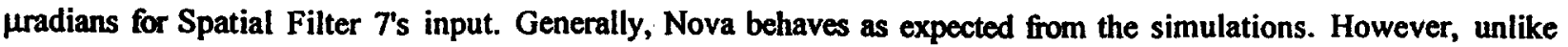
Beamlets data sets, some of the high power Nova tests indicate that the near-field modulation increases with tighter filtering (Fig. 7 ,right side). We suspect that the source terms in the model are insufficient. B-T gain calculations indicate that noise terms produced by optics in the preamplifier section of Nova, those upstream from the $31.5 \mathrm{~cm}$ and $46 \mathrm{~cm}$ amplifier stages, can undergo large nonlinear power growth. Furthermore, such noise terms cannot practically be removed from the beam by Nova's spatial filters. Magnifying filters like Nova's reduce the spectral angles of the beam passing through them, making it difficult to remove early-stage noise without seriously disturbing the irradiance profile of the beam. This noise gets "clipped" at the tight transport filter, giving rise to "Gibbs" ringing or modulation in the near field irradiance profile, at spatial frequencies characteristic of the pinhole cut-off angle. Nova's near field data at powers above 8 TW shows this effect, as "hot" spots develop in the beam with a characteristic scale size of $\lambda / \theta \sim 1-\mathrm{cm}$ with a $100-\mu$ radian pinhole angle.

\section{SUMMARY}

In the Beamlet tests we measured the near-field profile in the plane where the crystals are located, five meters past the output lens of the transport filter. As was observed in the Nova tests, for $\Delta \mathrm{B}$ values less than 1.8 radians, the contrast ratio has a value of $8 \%(0.08)$, which, within error, is independent of the pinhole size. As $\triangle B$ increases above 1.8, the system with the smaller $130 / 100 \mu$ radians pinholes shows a substantially slower rise in contrast ratio than those with the larger 200/200 $\mu$ radians pinholes. This result is in agreement with modeling calculations (but contradicts results obtained on Nova), indicating that there are no unidentified large sources of noise on Beamlet. From the data we conclude that Beamlet with $200 \mu$ radians pinholes in the cavity and transport filter can safely operate up to a B value in the booster amplifier of 1.8. If the pinholes can be reduced to $130 \mu$ radians for the final pass through the cavity and $100 \mu$ radians in the transport filter, the safe operating limit appears to be at a B value of 2.4. Although not shown here, the PROP92 simulations indicated the likelihood of significant beam filamentation in the last booster slab, or in $\mathrm{L3}$ at a $\triangle \mathrm{B}$ (in booster slabs) above 2.2 radians with $200 \mu$ radian cavity pinholes, or above 2.8 radians with $130 \mu$ radian pinholes. Figure 4 indicates that this filamentation onset occurs at a fluence contrast of $\sim 0.15$, or $15 \%$. The temporal peak contrast (not shown) at this point is $0.4 \sim 0.5$ depending on the particular simulation. The $99 \%$ percentile modulation can exceed 4 to 1 and the peak intensities can be 10 times the spatial average, resulting in intensities exceeding $40 \mathrm{GW} / \mathrm{cm}^{2}$ in the last booster slab or in L3. Catastrophic beam filamentation and collapse will follow in a few $\mathrm{cm}$ distance causing multiple damage tracks in the bulk material and at the exit surface. Since this damage occurs prior to the transport filter, "safe" operation requires that we stay below these limits with a certain margin for operational variations.

On Nova, with the presently used spatial filter pinholes and beam uniformity (or lack thereof), peak $\Delta B$ 's should be restricted to a redline value of 1.8 radians and normal operation should be $\sim 90 \%$ of this value, or 1.6 radians. For example, $1 \mathrm{~ns}$ redline operation should be limited to $6 \mathrm{TW} / 6 \mathrm{~kJ}$ and normal operation to $5 \mathrm{TW} / 5 \mathrm{~kJ}$. Simulations and experiments at these powers and $\Delta \mathrm{B}$ levels show that the near-field irradiance fluctuations are modest; i.e., the contrast ratio of the beam's irradiance prior to filtering is -0.1 , and after filtering is about half this value. At these reduced powers and energies, the contrast ratio appears to be independent of pinhole size for acceptance half angles between $100 \mu$ radians and $200 \mu$ radians; i.e., pinhole diameters of $1.5 \mathrm{~mm}$ to $3 \mathrm{~mm}$ in the penultimate filter and $1.8 \mathrm{~mm}$ to $3.6 \mathrm{~mm}$ in the final filter.

Minimizing the $1 \omega$ irradiance fluctuations at the frequency conversion crystals is extremely important to control beam filamentation at $3 \omega$ in the target chamber or focusing vessel final optics, and depends on good beam uniformity and the optimum pinhole sizes in cavity and transport filters. If the operation of Nova is restricted to $\Delta B$ 's of less than 1.8 radians, it should provide a reasonable margin against damage. On Beamlet and the NIF, a $\Delta B$ 
of 2.4 radians in the booster amplifiers could be tolerated (with acceptable margin to be determined), with 130 or $100 \mu$ radian pinholes. These actions should reduce the damage and filamentation threat to the conversion crystals and UV optics.

\section{ACKNOWLEDGMENTS}

The authors thank the Beamlet and Nova operations teams for their work in these experiments. This work was performed under the auspices of the U.S. Department of Energy by Lawrence Livermore National Laboratory under Contract No. W-7405-Eng-48.

\section{REFERENCES}

1. R. Sacks, W. Williams, M. Henesian, C. Orth, S. Haney, J. Trenholme, J. Auerbach, and J. Lawson, "Propagation modeling in two transverse dimensions of the NIF baseline performance", presented to the Second Annual International Conference on Solid State Lasers for Application to Inertial Confinement Fusion, October 22-25, 1996, Paris, France.

2. C. Widmayer, J. Auerbach, R. Ehrlich, M. Henesian, J. Hunt, J. Lawson, D. Milam, P. Renard, D. Speck, P. Wegner. T. Weiland, W. Williams, C. Wolfe, and B. Van Wonterghem, "Producing National Ignition Facitlity (NIF) quality beams on the Nova and Beamlet lasers," $12^{\text {th }}$ Topical Meeting on the Technology of Fusion Energy, Reno, Nevada, June 16-20, 1996.

3. J. Lawson, L. Seppala, I. Smith, and C. Thompson, "Beamlet Schlieren Diagnostic and Experiments," presented to the Second Annual International Conference on Solid State Lasers for Application to Inertial Confinement Fusion, October 22-25, 1996, Paris, France.

4. R. Sacks, M. Henesian, S. Haney, and J. Trenholme, "The PROP92 Fourier Beam Propagation Code," to be published in the Inertial Confinement Fusion ICF Quartery Report, April-June, 1997, Volume 7, Lawrence Livermore National Laboratory, UCRL-LR-105821-97-3 (1997).

5 B. Van Wonterghem, J. Murray, J. Auerbach, S. Burkhart, J. Caird, M. Henesian, F. Penko, and P. Wegner, "High Fluence $1 \omega$ Performance Test using 20 ns Ignition Shaped Pulses on the Beamlet Prototype Laser," presented to the Second Annual International Conference on Solid State Lasers for Application to Inertial Confinement Fusion, October 22-25, 1996, Paris, France.

6. W. Williams, K. Manes, J. Hunt, P. Renard, D. Milam, and D. Eimerl, "Modeling of Self-Focusing Experiments by Beam Propagation Codes," Inertial Confinement Fusion ICF Quartery Report, Oct-Dec, 1995, Volume 6, Lawrence Livermore National Laboratory, UCRL-LR-105821-96-1 (1996).

7. J. Hunt, K. Manes, and P. Renard, "Hot images from obscurations," Aplied Optics 32, 5973-5982 (1993).

8. J. Lawson, D. Aikens, R. English, and C. Wolfe, "Power spectral density specifications for high-power laser systems," proceedings of Specification, Production, and Testing of Optical Components and Systems, May 1316, 1996, Glasgow, Scotland, UK, SPIE Proceeedings, Vol. 2775, 345-356 (1996).

9. M. Henesian, S. Haney, M. Thomas, and J. Trenholme, "Modeling for Deformable Mirrors and the Adaptive Optics Optimization Program," presented to the Second Annual International Conference on Solid State Lasers for Application to Inertial Confinement Fusion, October 22-25, 1996, Paris, France. 


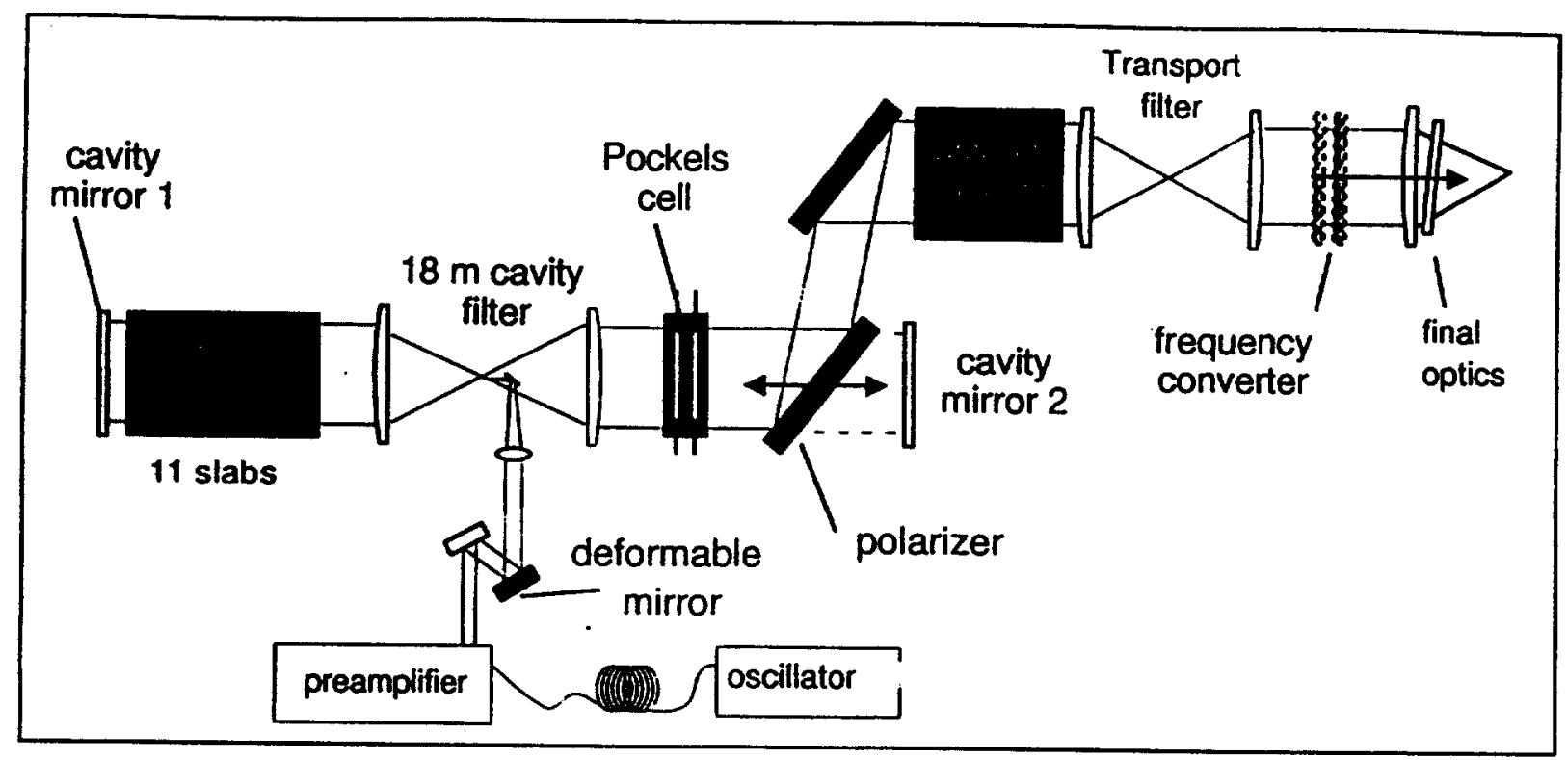

Figure 1. Beamlet and NIF 11-0-5 baseline designs are very similar. Beamlet uses 18-m spatial filters, whereas NIF will use $23.5-\mathrm{m}$ cavity and $60-\mathrm{m}$ transport filter lengths. Beamlet injects beam into cavity filter pinhole plane as shown, whereas NIF will use the transport filter for beam injection.

Beamlet: 5 time slices

NIF: 8 time slices

mlet-200ps-135-3tw. Beamlet,DFM,3-TW/200-ps,Disk Type 16,135-wr SF redksr52 107: nil slandord 20 model boseline orctilecture creoled 3-13-96
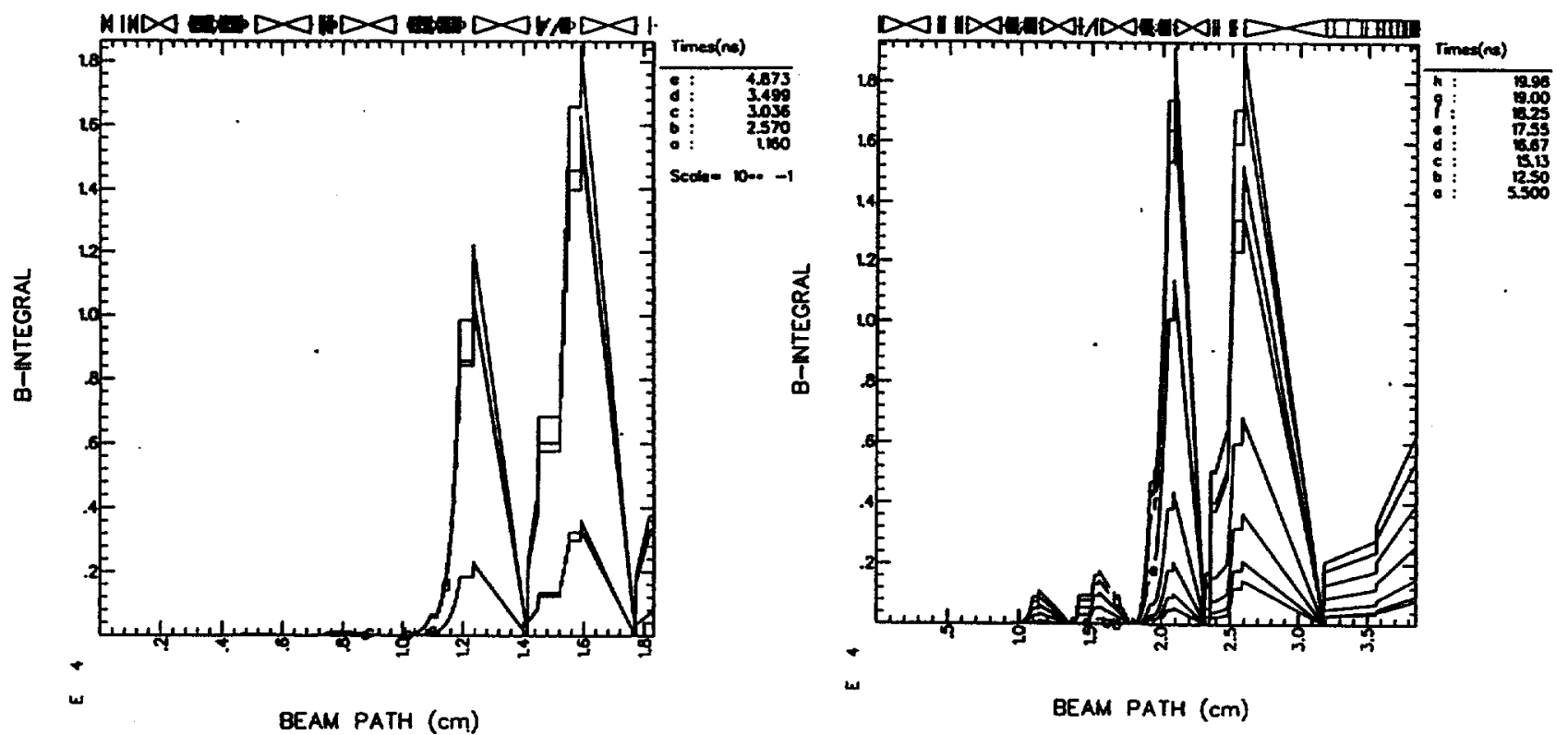

Figure 2. B-integral plots for a 200-ps duration pulse on Beamlet (left) with unpumped booster amplifiers, and for a 20-ns shaped pulse for NIF (right). $\triangle B$ in the booster amplifier sections are similar for the two cases. 


\section{Simulated Injection Beam}

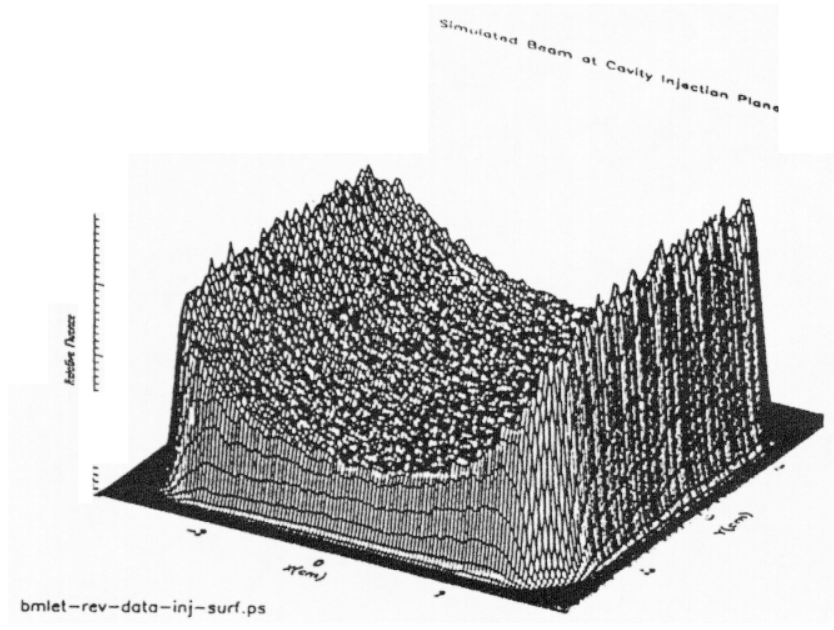

Contrast from simulations:

$0.0478(4.78 \%)$

\section{Measured Injection Beam}

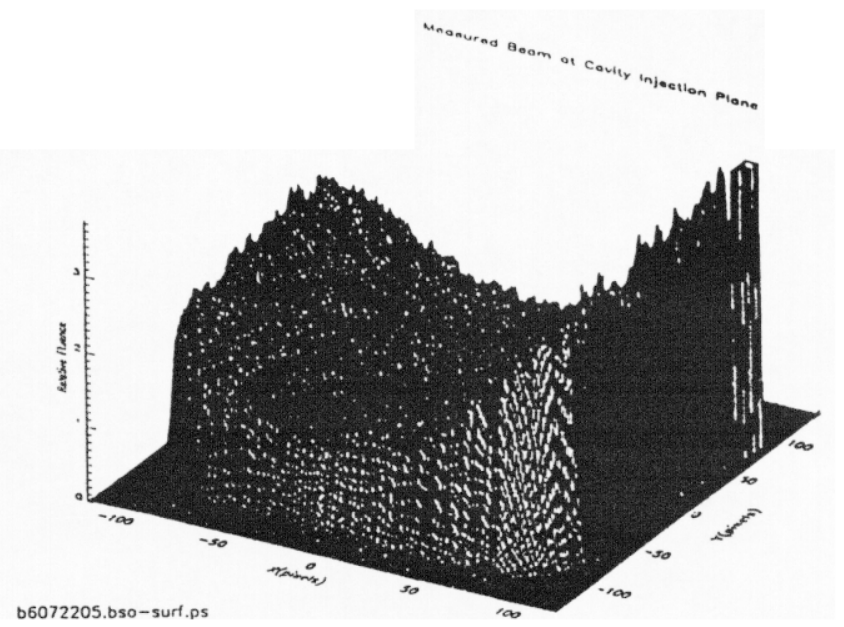

Measured contrast from equivalent plane film data (ref. b6081916) : $0.0455(4.55 \%)$

Figure 3. Front-end model for Beamlet generates an injection beam profile for the 4-pass main cavity that is very close to measured shape and fluence contrast.
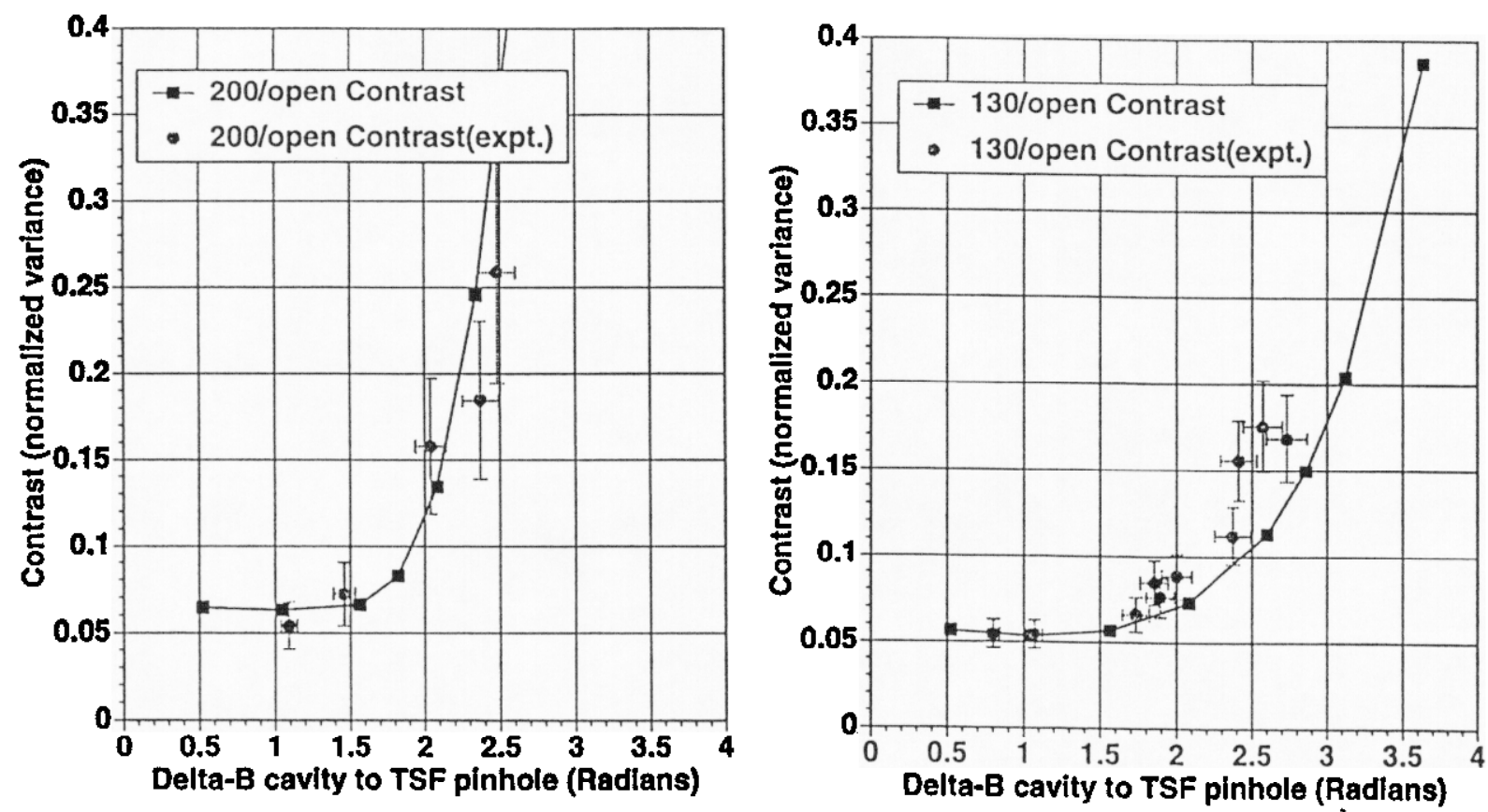

Figure 4. Contrast measurements and predictions for Beamlet with open transport pinhole that allows us to measure the nonlinear growth of modulation for the unpumped booster amplifier stage. 

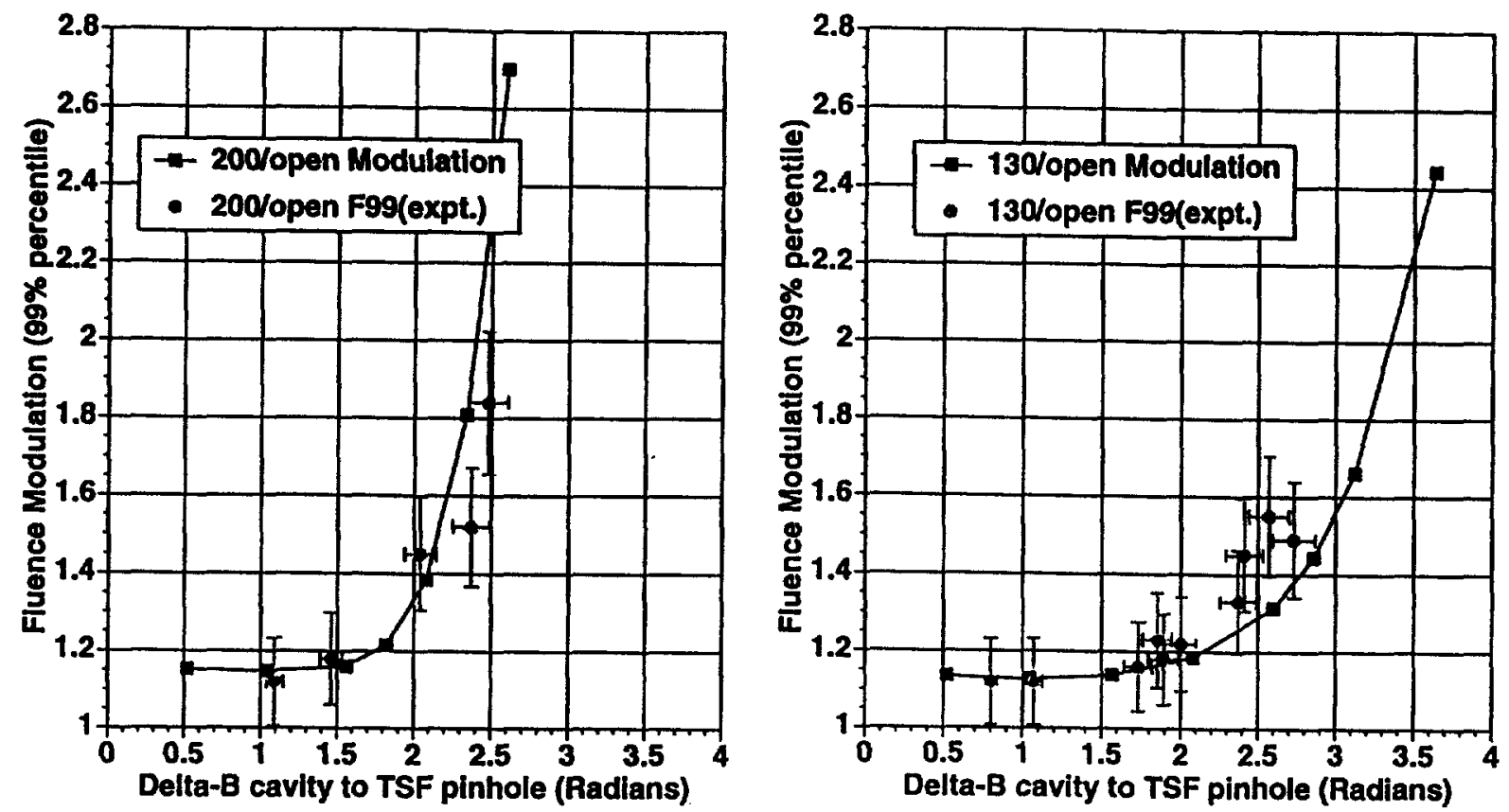

Figure 5. Fluence modulation $\left(F_{99}\right)$ at the $99^{\text {th }}$ percentile of the fluence histogram with open transport pinhole verifies the damage threat above $\Delta B \sim 2.2$ with 200/open and $\Delta B \sim 2.8$ with 130/open configurations.
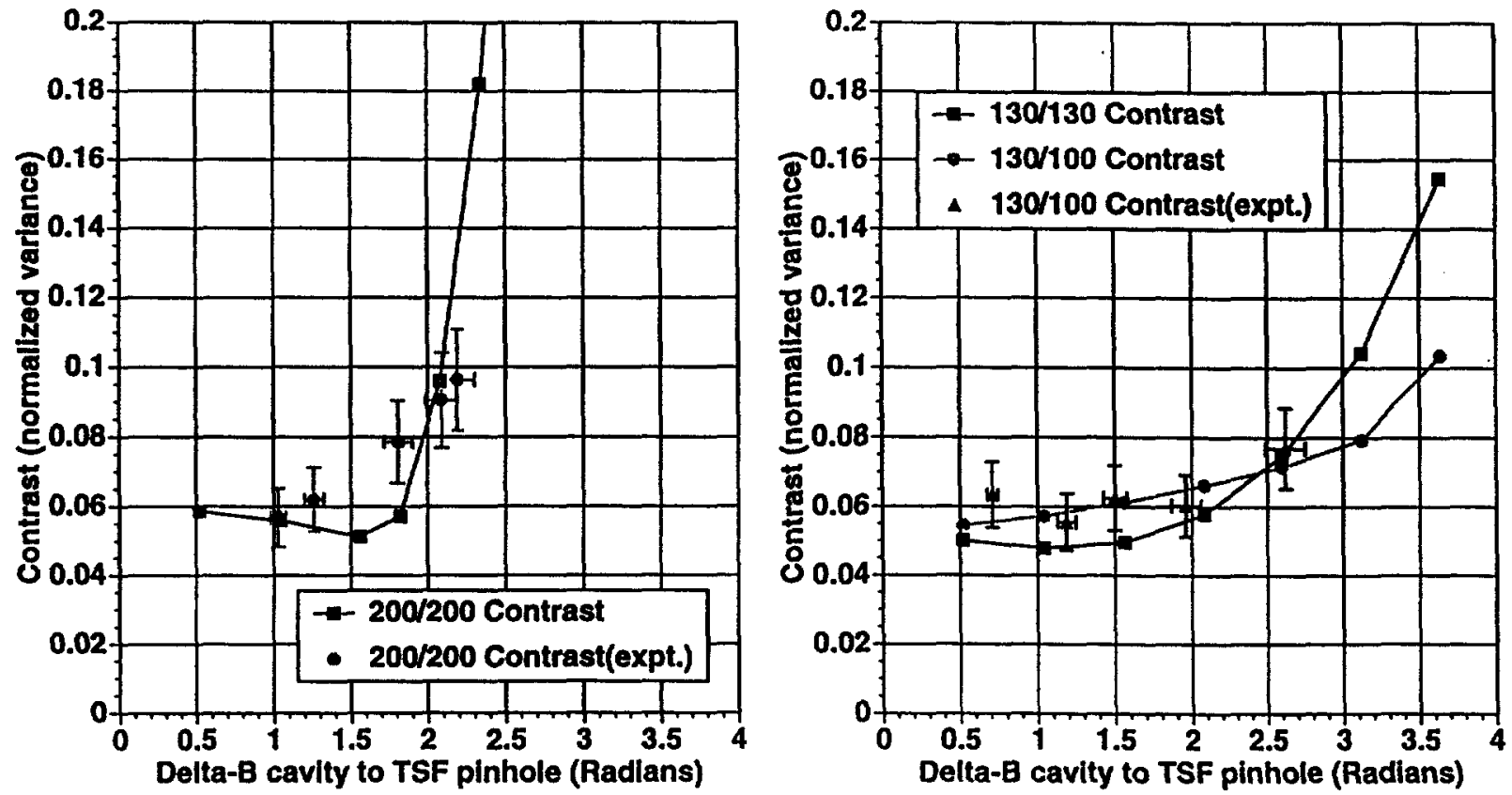

Figure 6. Transport spatial filtering is used to control the near field modulation and damage threat to down stream optics. Contrast shown for $200 / 200-\mu$ radian and $130 / 130,130 / 100-\mu$ radian transport pinholes. 


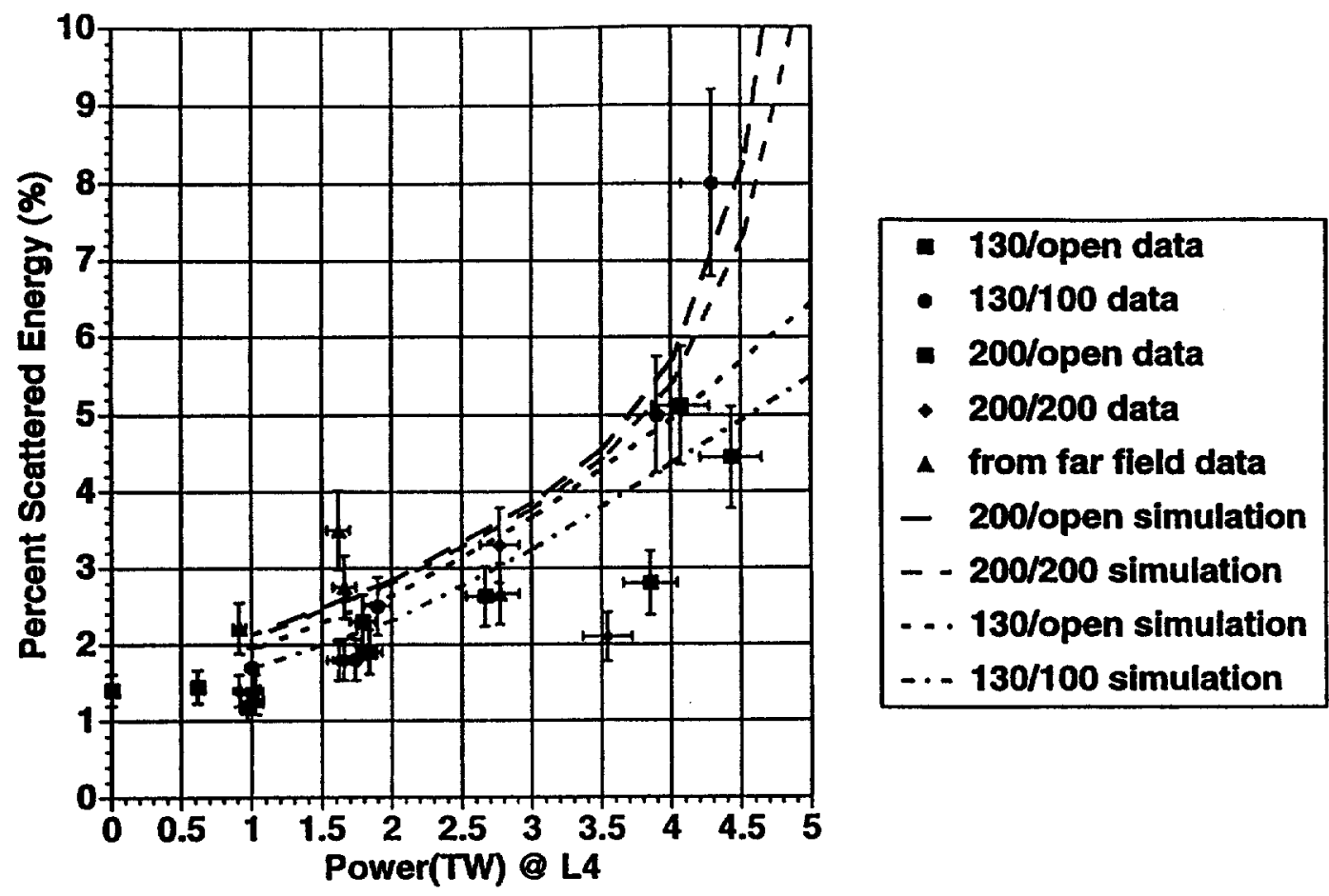

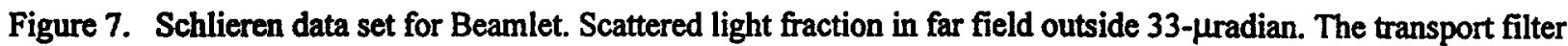
pinhole size has less effect on scattered energy than the cavity pinholes. Energy rises rapidly after $\triangle B \sim 2$ radians at $3.8 \mathrm{TW}$ power.
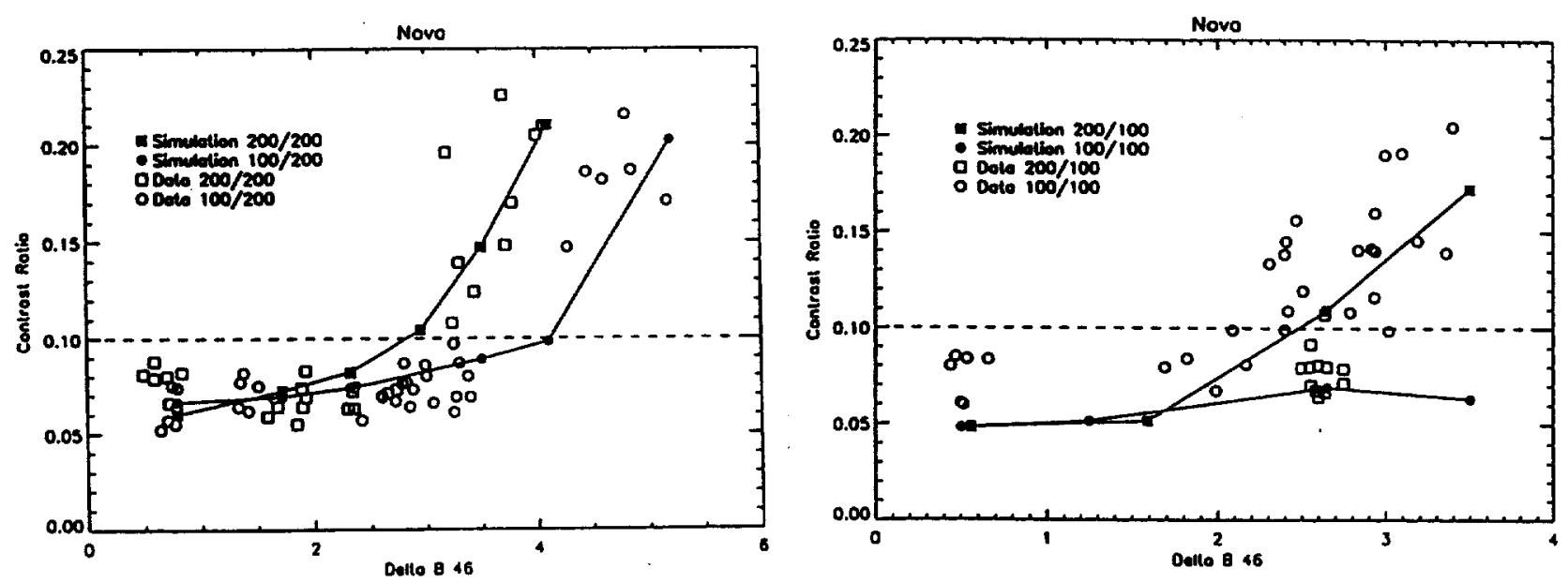

Figure 8. Contrast ratio vs. $\Delta \mathrm{B}$ in $46-\mathrm{cm}$ disk amplifier stage for two pinhole configurations on Nova. Current standard pinholes are 200- $\mu$ radian for SF6 and 200- $\mu$ radian for SF7. Plot on right shows Nova results with $100-\mu$ radian transport pinhole SF7, and 100 or $200-\mu$ radian SF6 pinhole. 


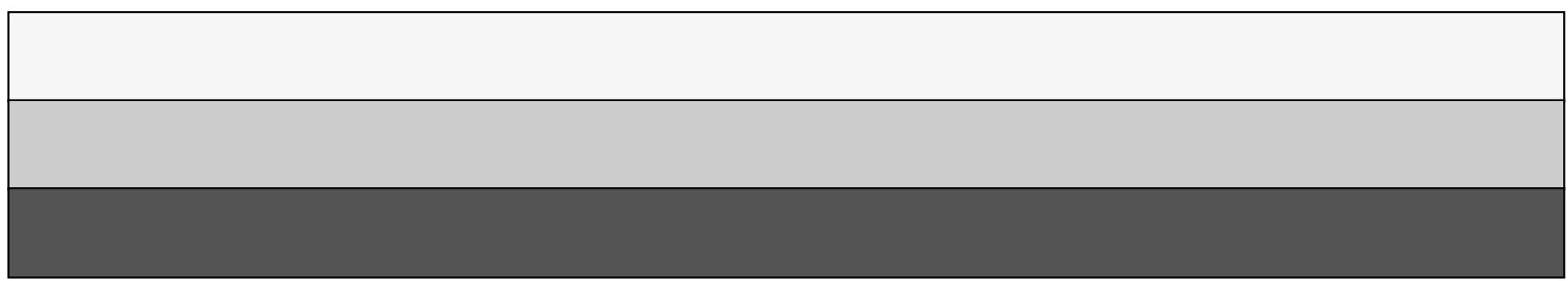

